\title{
Parallel Information Processing in Motor Systems: Intracerebral Recordings of Readiness Potential and CNV in Human Subjects ${ }^{\dagger}$
}

\author{
Ivan Rektor \\ 1st Department of Neurology, Masaryk University, St.. Anne's Hospital, \\ Pekařská 53, 65691 Brno, Czech Republic \\ This article is in memory of Simon Brailowsky
}

\section{SUMMARY}

We performed intracerebral recordings of Readiness Potential (RP) and Contingent Negative Variation (CNV) with simple repetitive distal limb movement in candidates for epilepsy surgery. In 26 patients (in Paris), depth electrodes were located in various cortical structures; in eight patients (in Brno), in the basal ganglia and the cortex. RPs were displayed in the contralateral primary motor cortex, contralateral somato-sensory cortex, and bilaterally in the SMA and the caudal part of the anterior cingulate cortices. CNVs were recorded in the same cortical regions as the RP, as well as in the ipsilateral primary motor cortex, and bilaterally in the premotor fronto-lateral, parietal superior, and middle temporal regions. In the basal ganglia, the RP was recorded in the putamen in six of seven patients, and in the head of the caudate nucleus and the pallidum in the only patient with electrodes in these recording sites. We suggest that our results are consistent with a long-lasting, simultaneous activation of cortical and subcortical structures, before and during self-paced and stimulus-triggered movements. The particular regions that are simultaneously active may be determined by the task context.

Corresponding author:

Prof. Dr. Ivan Rektor, CSc

Masaryk University, Hospital Sv. Anna

65691 Brno Czech Republic

Phone: + 420543182623 ; Fax: +420 543182624

e-mail: irektor@med.muni.cz
KEYWORDS

cognitive, SEEG, motor regulation, CNV, MASP, readiness potential, putamen

\section{INTRODUCTION}

A variety of sustained potentials can be recorded in human subjects during different cognitive activities. On the scalp, the amplitude and topography of slow potential shifts depend on the memory load, cognitive strategy, and modality of presentation (Lang et al., 1991). Several slow waves have been observed, both before and during movement.

Movement-related Readiness Potentials (RP, or Bereitschaftpotential) precede self-paced voluntary movements by 1 to 2 seconds. RP is composed of several waves. The Contingent Negative Variation (CNV) is a complex waveform comprising at least two waves interposed between potentials that are evoked by stimulus signals. The second wave is thought to share critical features with motor preparation (Brunia \& Damen, 1988). RP and CNV are believed to be related to various cognitive constants (Libet, 1985, Brunia \& Damen, 1988). We performed intracerebral recordings of RP and CNV with a simple, repetitive distal-limb movement. The results concerning the topography of the generating structures have been published elsewhere 
(Rektor et al., 1994; Lamarche et al., 1995; Rektor et al.1998a; Rektor et al., 1998b). In this paper we will discuss the results of the cortical and subcortical recordings from the point of view of brain network operations that are linked with movement preparation and execution.

\section{PATIENTS AND METHODS}

Two groups of patients suffering from drugresistant partial epilepsy were studied. Stereoelectroencephalography (SEEG) was performed with implanted intracerebral electrodes. In Paris (Hôpital Ste Anne, Service de Neurochirurgie; INSERM, U 97), 26 patients had orthogonal cortical electrodes. The Readiness Potential (RP) was tested in 17 patients, Contingent Negative Variation (CNV) in 14 patients, and both protocols in 7 patients. In Brno (Centre for Epilepsy, St. Anne's Hospital), eight patients had electrodes in the basal ganglia and in the cortex. In addition to the orthogonal electrodes, these patients also had one diagonal electrode, implanted stereotactically, that reached the amygdala and hippocampus via the basal ganglia. In seven patients, the basal ganglia contacts were located in the putamen, and in one patient, in the head of caudate nucleus and in the pallidum. Here we report the results of RP recordings in the basal ganglia in this group. The cortical recordings were performed in temporal, frontoorbital, and opercular regions in this group with suspected temporal lobe epilepsy. RP was not produced in these cortical regions.

Two paradigms were tested: RP and CNV. Slightly modified classical testing protocols were used (Kornhuber \& Deecke, 1965; Deecke, 1985; Walter et al., 1964). In the Paris group, both tests were performed, and RP was tested in the Brno group. We investigated slow potentials appearing before (RP and $\mathrm{CNV}$ ) and during (Movement Accompanying Slow Potential, MASP) a simple repetitive distal limb movement, namely with a wrist or a foot flexion. (Fig.
1). The movement was self paced in the RP protocol and was triggered by an external signal in the CNV protocol. In the latter test, auditory signals were used for both the warning and the imperative stimuli, with an interstimulus interval of 1.5 and $3.0 \mathrm{sec}$.

DIXI intracerebral 5-15 contact stainless steel and platinum electrodes were used for the recordings (electrode diameter $=0.8 \mathrm{~mm}$, contact length $=2 \mathrm{~mm}$, distance between contacts $=1.3$ $\mathrm{mm}$ (Fig 1). The stereotactic method of Bancaud and Talairach (1965) was used for orthogonal electrodes. The electrodes were introduced into sites corresponding to the electroclinical characteristics of each patient's seizures. The recordings from contacts with EEG pathology and from contacts located in lesioned areas were excluded from the evaluation.

Data acquisition and averaging were performed using a Nihon Kohden Neuropack 4 and 8 set, the band was 0,01 to $500 \mathrm{~Hz}$ for the intracerebral recordings and 200 to $3 \mathrm{kHz}$ for the electromyography (EMG). The analysis window was $5 \mathrm{sec}$. All recordings were taken against a biauricular reference. Every set of four or eight channels (three or seven intracerebral, one muscle trigger) was recorded on-line, visually evaluated, and artifacts (blinking, other movements, etc.) were rejected. Movements that started earlier than $5 \mathrm{sec}$ after the previous one were automatically rejected from the averaging process. In every session, the number of trials was 40 to 60 . The presence or absence of slow potentials, their shape, polarity, and duration were analyzed off-line. No recordings of slow potentials from regions with MRI lesions and pathological EEG activity were taken. More details concerning patients and methods have already been published (Rektor et al., 1994; Rektor et al., 1998a; Rektor et al., 1998b; Lamarche et al., 1995).

\section{RESULTS}

Figures 2 and 3 show that the cortical distribution of RP, CNV, and MASP overlapped 


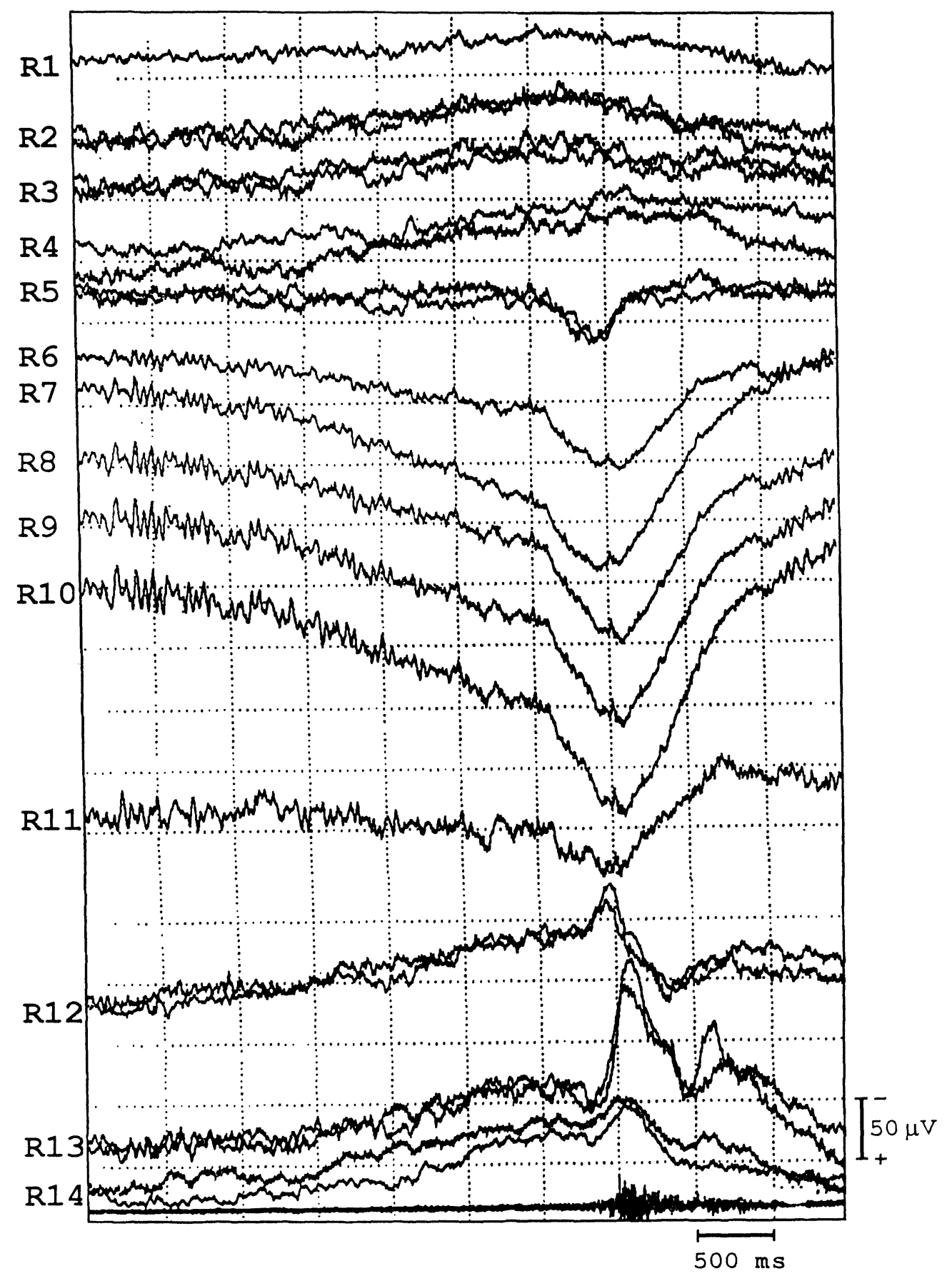

Fig. 1: Readiness Potential (RP) and Movement Accompanying Slow Potential (MASP) recorded on consecutive leads on an electrode passing from the post-central cortex ( $R$ 14) through the primary motor cortex ( R13$R 11$; phase reversal between $R 11$ and $R$ 12) to SMA (R5-R1;phase reversal between $R 4$ and $R$ 5). EMG: the contralateral (right) hand movement. Results of consecutive tests are superimposed in most cortical contacts (R2-R5 and R12-R14), indicating that MASP is a reproducible phenomenon. Notice variable morphology of MASP, which is displayed both as multiphasic (R12,13) and as monophasic. Even when displayed together, MASP is not a simple component of RP. When recorded on consecutive contacts, the development of RP- and MASP- slopes and their phase reversals differed. Reprinted from: Rektor I. et al. Electroencephalogr clin Neurophysiol, 1998; 107: 277-286, with permission from Elsevier Science. 

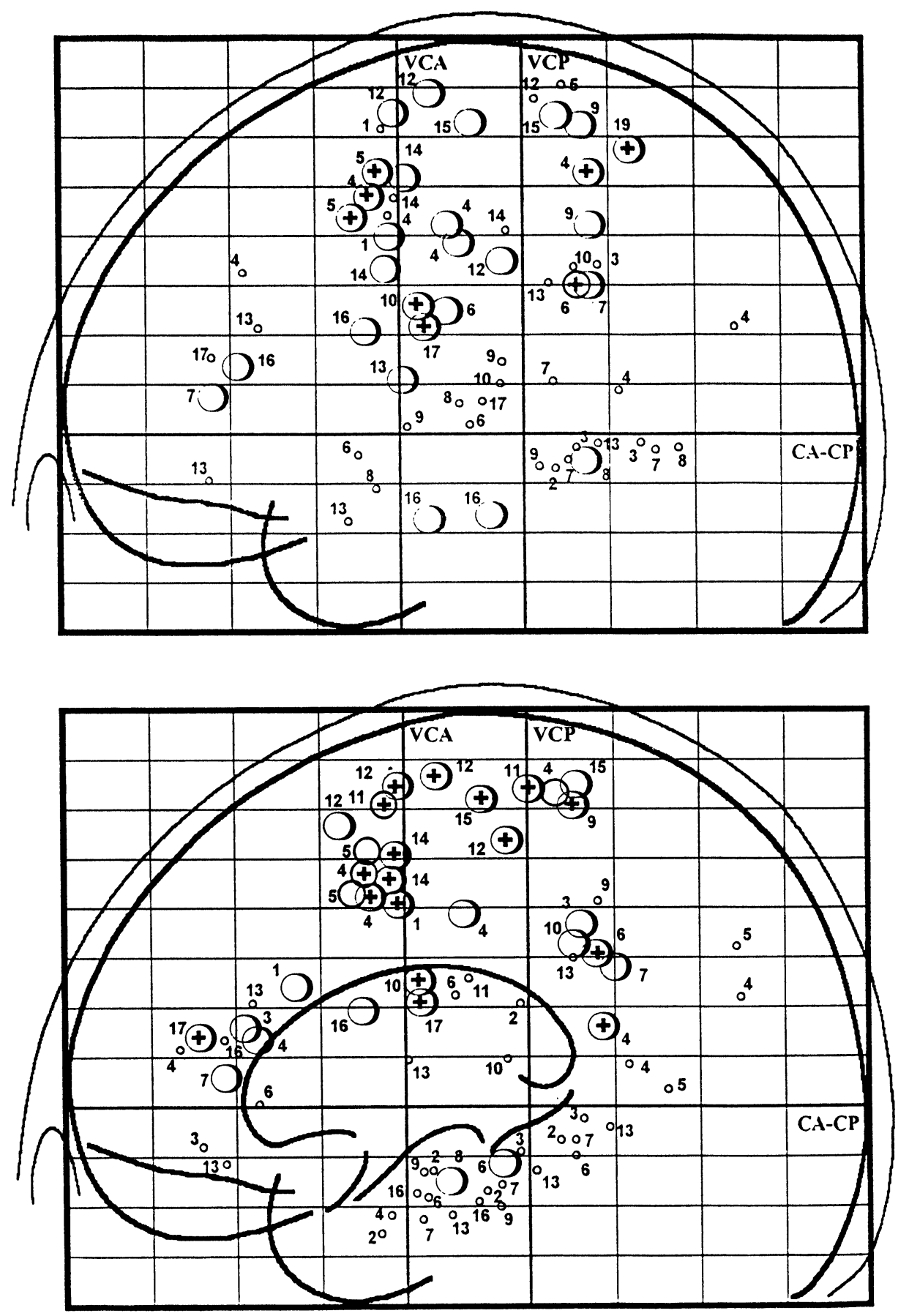

Fig. 2: The cortical recording sites in the Readiness Potential paradigm. Upper part: Lateral view of the brain. Lower part: Sagittal aspect. In the supracallosal part the internal contacts of multilead electrodes mostly reached the mesial wall. In the lower part of the diagram some of the recording points correspond to sites located more laterally. This schema presents the results in one or in both hemispheres. The numbers correspond to the individual patients. The large circles show the MASP occurrence. They are crossed if a Readiness Potential preceded the MASP. The small circles indicate electrodes which recorded no slow potential. Reprinted from: Rektor I. et al. Electroencephalogr clin Neurophysiol, 1998; 107: 277 - 286, with permission from Elsevier Science. 

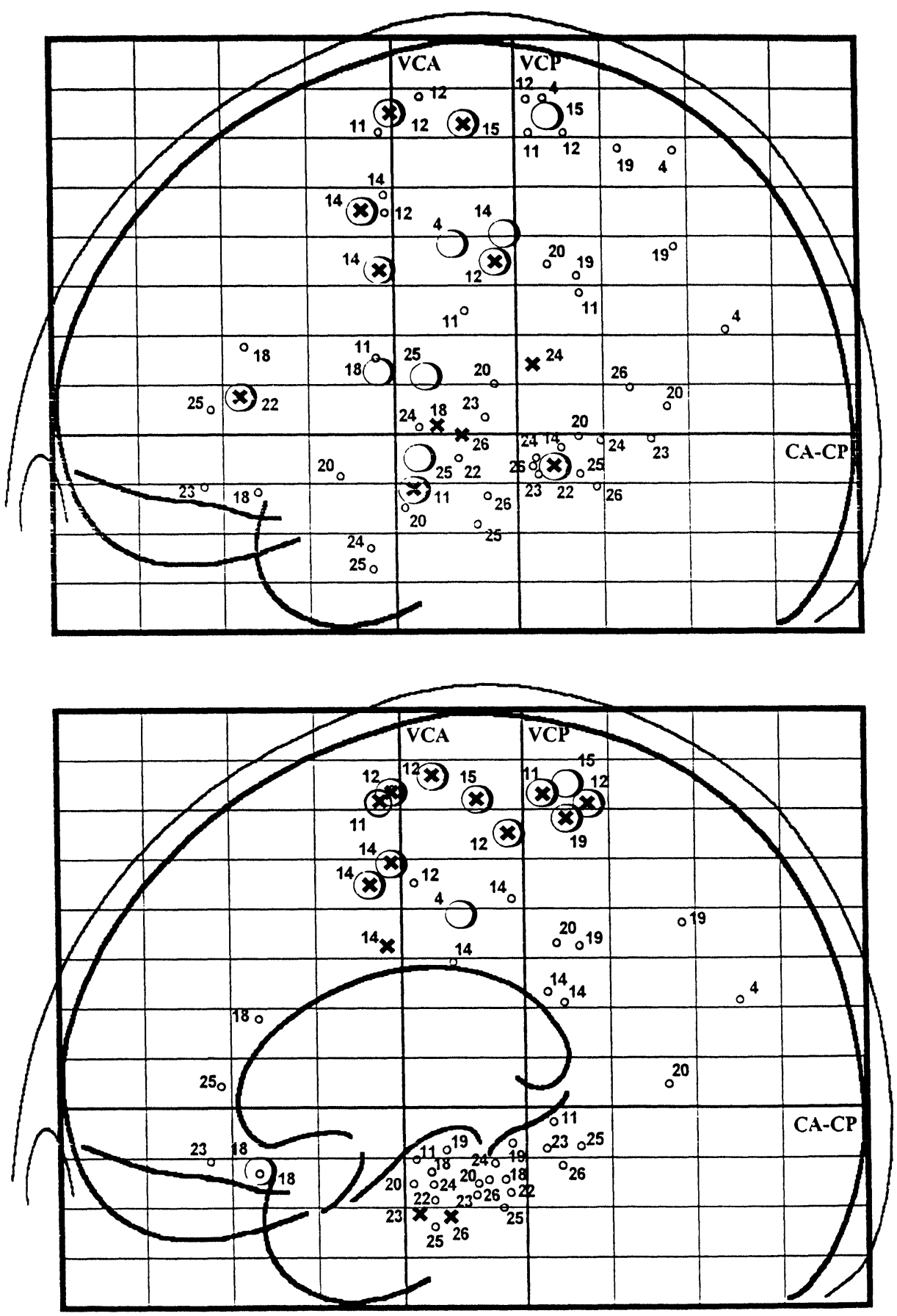

Fig. 3: Cortical recording sites in the Contingent Negative Variation Paradigm. Upper part: Lateral view of the brain. Lower part: Sagittal aspect. In the supracallosal part, the internal contacts of multilead electrodes mostly reached the mesial wall. In the lower part of the diagram, some recording points correspond to sites located more laterally. This schema presents the results in one or in both hemispheres. The numbers correspond to individual patients. The large circles show the MASP occurrence. The circles are crossed if CNV preceded MASP. The small circles indicate electrodes that recorded no slow potential. The non-circled crosses represent sites with CNV, but with no movement-accompanying slow potential. Reprinted from: Rektor I. et al. Electroencephalogr clin Neurophysiol, 1998; 107: 277-286, with permission from Elsevier Science. 
in some areas and differed in others (Note: Figs. 2 and 3 are purely schematic. Adding the coronal plan would be misleading because of the very variable depth of cortical sulci. In some cases, only the most superficial contact was located in the cortex; in other cases the cortex could be explored also by some contacts in the central segment of the electrode ).

Six cortical regions with simultaneously displayed RPs were observed: the contralateral primary motor cortex, contralateral somatosensory cortex, and bilaterally, the SMA and the caudal part of the anterior cingulate cortices (Rektor et al., 1994, Lamarche et al., 1995). The cortical recordings performed in Brno did not contain regions with RP. The absence of RPs was common for both the Paris and Brno groups. In the latter group, basal ganglia were explored as well. The putamen was explored in seven patients, and the head of nucleus caudatus and the pallidum internum were studied in one patient. RP was observed in the pallidum and in the caudate nucleus and was also recorded in putamen in six out seven patients. RPs were displayed bilaterally, preceding the movement onset by 750 to $1900 \mathrm{~ms}$. The shape of the RP recorded in the basal ganglia was similar to that recorded on the scalp. The potentials accompanying the movement were also present in the explored subcortical structures. Their electrophysiological properties, for example, the polarity, differed from RP indicating separate generators.

The cortical distribution of the CNV-like potentials was broader. In addition to the cortical regions in which we observed the RPs, the CNVs were also found in the ipsilateral primary motor cortex, in the premotor frontolateral cortices bilaterally, in the parietal cortices bilaterally (mostly in Brodman's area 5 and 7), and in the superior and middle temporal gyri bilaterally (Lamarche et al., 1995, Rektor et al., 1998a).

The potentials recorded during the execution of the movement (MASP) either followed the potentials preceding the movement, or they occurred alone without the presence of a pre- movement potential (Fig. 1). The MASP were broadly distributed in motor, supplementary motor, pre-motor, pre-frontal, mid-temporal, somatosensory, superior parietal and cingulate cortices. A difference between the cortical distribution of the self-paced MASP and the externally cued MASP was seen. In the primary motor cortex, MASP was present contralaterally to the movement in the RP paradigm and bilaterally in the CNV protocol. (In all other areas the MASP occurred bilaterally). The cingulate cortex was heavily involved bilaterally in the self-paced movements but rarely in the cued movements. The major involvement of the cingulate gyrus was particularly striking with the absence of any slow potentials in the mesial temporal lobe structures in both protocols. More details concerning the results have already been published (Rektor et al.1994, Rektor et, 1998a, Rektor et al., 1998 b, Lamarche et al., 1995).

\section{DISCUSSION}

We observed that variations in movementrelated potentials occur simultaneously in multiple cortical and subcortical structures. According to the model designed by Alexander (1994), a motion is based on the sequential activity of structures, arranged in a cortico-subcortico-thalamo-cortical circuitry, that are modulated by several sub-cortical loops. This model was criticised recently (Parent \& Cicchetti, 1998), nevertheless the basic principles are commonly accepted.

Our results indicate that in addition to a sequential processing of information, a simultaneous, long activation of cortical and subcortical structures, lasting hundreds of milliseconds to seconds, also occurs. This observation is in accordance with the growing awareness that the anatomy and physiology of the motor system is not consistent with an exclusively sequential model of motor command and control. This result fits better with the concept that sensori- 
motor processing is an operation distributed in a parallel manner, conducted simultaneously at several brain sites (Houk et al., 1993).

The slow waves comprise several elements (for example, iCNV and $\mathrm{tCNV}$, or BP and NS in the RP) reflecting the notion that the slow activation is a composite phenomenon. It is evident that slow potentials do not only reflect the neuronal discharges generating a movement and the arrival of sensory feedback information related to the movement itself. Prolonged voluntary muscle contraction alone does not cause any cerebral negativity, either before or after movement, if the action is not goal directed, or has no motivational significance (Tecce et al., 1984, quoted in: Birbaumer et al., 1990). But at the same time, the appearance of RP, CNV, and MASP is not indispensable for preparing and performing a movement. This conclusion was demonstrated in our studies and also in studies of cases with lesions of basal ganglia, in which slow potentials did not appear on the scalp while the movement was being performed (Fève et al., 1991, Deecke, 1985). The appearance of slow waves was determined more by the task condition preceding the movement than by the movement itself, for example, in the primary motor cortex. On the contrary, the cortical distribution of RP is not influenced by the type of movement performed by a given limb (compare identical distribution of RP with hand and wrist flexion in our studies and with finger flexion in the studies of Ikeda and colleagues (Rektor et al., 1994; 1998a; Ikeda et al., 1992).

The topography of simultaneously active cortical regions is task related. During identical movements, the areas with slow activation were different, depending on the kind of pacing. The slow potentials in the self-paced paradigm were present in the primary motor cortex, only contralateral to the movement, but they were bilateral in the CNV protocol. The cingulate cortex was frequently involved in the self-paced movements but rarely in the cued movements. In the self-paced protocol, only the regions known to be involved in motor activity were involved: contralateral motor, somatosensory, bilaterally supplementary motor area, and bilaterally, the caudal part of anterior cingulate cortex and in the basal ganglia. In the CNV protocol with auditory stimulation, the slow waves (CNVs) appeared beside the motor and premotor regions and also in the acoustic temporal zone (Lamarche et al., 1995).

It was interesting to observe the wide distribution of cortical and subcortical involvement before and during a simple movement. The observation that a very elementary task like a wrist flexion was preceded and accompanied by a long-lasting activity in large areas of the brain might indicate that the regions involved are active simultaneously in several tasks.

\section{CONCLUSION}

1. A long-lasting, simultaneous activation of cortical and sub-cortical structures occurs in the motor system when a movement is prepared and executed.

2. Which regions are simultaneously active is dependent on the context of the task

\section{ACKNOWLEDGEMENT}

This research was supported by Grant GAÈR 309/98/0490. Research program MŠ CR 112801. The author thanks the co-authors of previous studies: M. Bareš, N. Bathien, P. Buser, A.Fève, D. Kubová, M. Lamarche, J. Louvel. The author thanks Tomáš Paus and Kees Brunia for important comments.

\section{REFERENCES}

Alexander GE. Basal ganglia-thalamocortical circuits: their role in control of movements. J. Clinical Neurophysiol 1994; 11: 420-431. 
Bancaud J, Talairach J, Bonis A, Schaub C, Szikla G, Morel P, Bordas-Ferrer M. La Stéréoencéphalographie dans l'Epilepsie. Paris: Masson, 1965; 321 pages.

Birbaumer N, Elbert T, Canavan AGM, Rockstroh B. Slow Potentials of the Cerebral Cortex and Behavior. (Review). Physiol Rev 1990; 70: 1-40.

Brunia CHM, Damen EJP. Distribution of slow brain potentials related to motor preparation and stimulus anticipation in a time estimation task. Electro-enceph clin Neurophysiol 1988; 69: 234-243.

Deecke L. Cerebral potentials related to voluntary actions: parkinsonian and normal subjects. In: Delwaide PJ, Agnoli A, (eds), Clinical Neurophysiology in Parkinsonism. Amsterdam: Elsevier, 1985; 91-105.

Fève A, Bathien N, Rondot P. Evolution des potentiels corticaux liés au movement chez les patients parkinsoniens, avant et après traitement par la levodopa. Neurophysiol Clin 1991; 21: 105-119.

Houk JC, Keifer J, Barto AG. Distributed motor commands in the limb premotor network. Trends in the Neurosciences 1993; 16: 27-33.

Ikeda A, Luders HO, Burgess RC, Shibasaki H: Movement-related potentials recorded from supplementary motor area and primary motor cortex. Brain 1992; 115: 1017-1043.

Kornhuber HH, Deecke L. Hirnpotentialänderungen bei Willkurbewegungen und passiven Bewegungen den Menschen: Bereitschafts-potential und reafferente Potentiale. Pflugers Arch 1965; 284: 1-17.

Lang W, Cheyne D, Kristeva R, Lindinger G,
Deecke L. Functional localisation of motor processes in the human cortex. Event-Related Brain Res 1991; (EEG Suppl. 42): 97-115.

Lamarche M, Louvel J, Buser P, Rektor I. Intracerebral recordings of slow potentials in a contingent negative variation paradigm: an exploration in epileptic patients. Electroen-cephalogr Clin Neurophysiol, 1995; 95: 268-276.

Parent A, Cicchetti F. The Current Model of Basal Ganglia Organization Under Scrutiny. Movement Disorders 1998; 13: 199-202.

Libet B. Unconscious cerebral initiative and the role of conscious will in voluntary action. Behav Brain Sci 1985; 8: 529-566.

Rektor I, Fève A, Buser P, Bathien N, Lamarche M. Intracerebral recording of movement-related Readiness potentials: an exploration in epileptic patients. Electroencephalogr Clin Neurophysiol 1994; 90: 273-283.

Rektor I, Louvel J, Lamarche M. Intracerebral recording of potentials accompanying simple limb movements: a SEEG study in epileptic patients. Electroencephalogr clin Neurophysiol 1998a; 107: 277-286.

Rektor I, Bareš M, Kubová D. Movement preparation in the basal ganglia. A SEEG Readiness potential study. Mov Disord 1998b; 13: (Suppl. 2) 101

Walter WG, Cooper R, Aldridge VJ, McCallum C, Cohen J. The contingent negative variation: an electrocortical sing of sensorimotor association in man. Electroencephalogr Clin Neurophysiol 1964; 7: 340-344. 

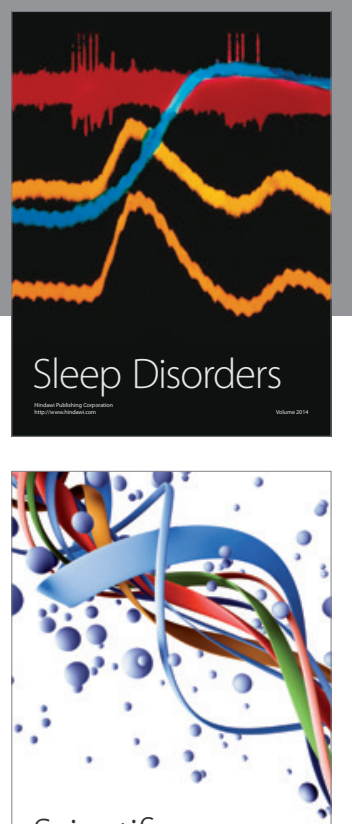

Scientifica
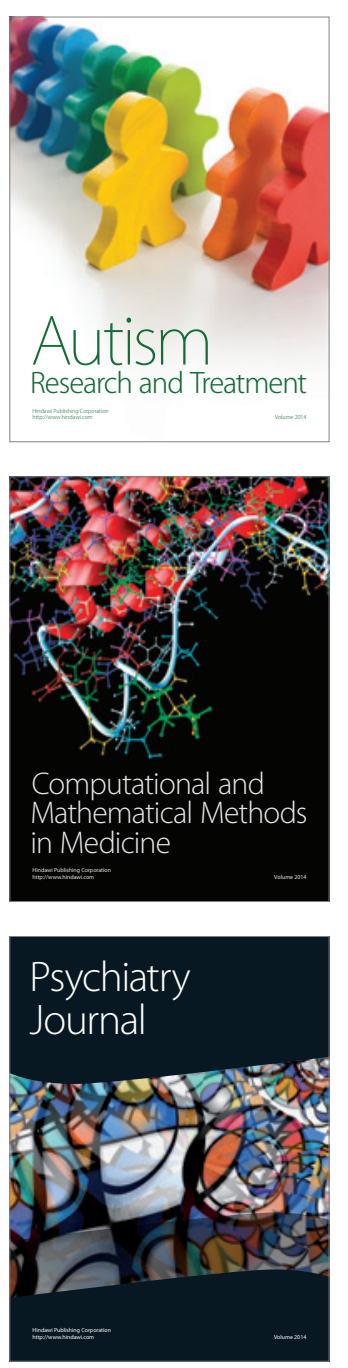
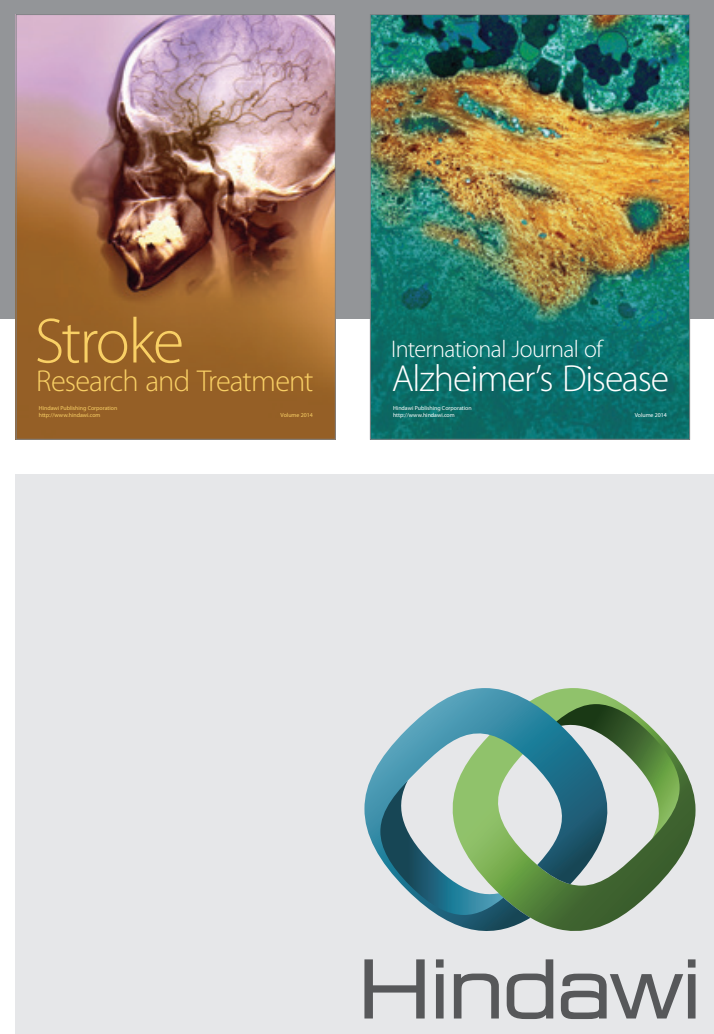

Submit your manuscripts at

http://www.hindawi.com
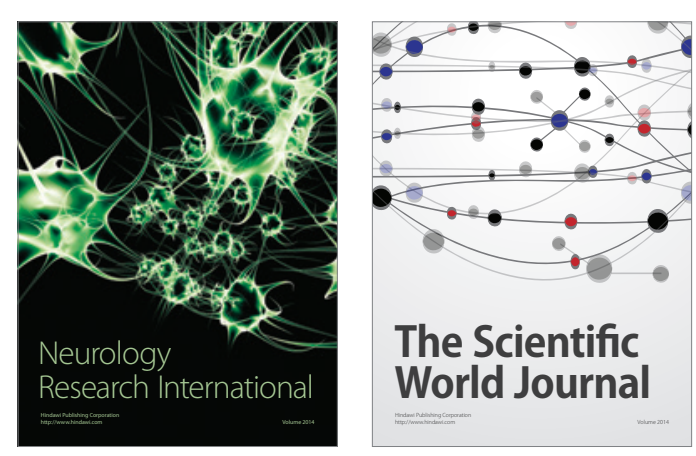

The Scientific World Journal

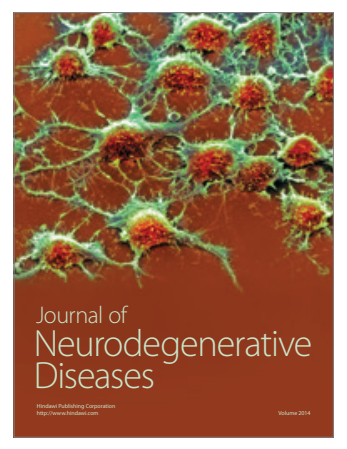

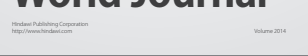

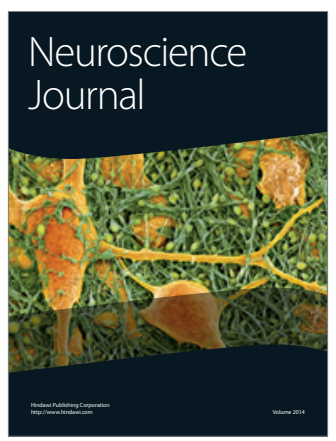

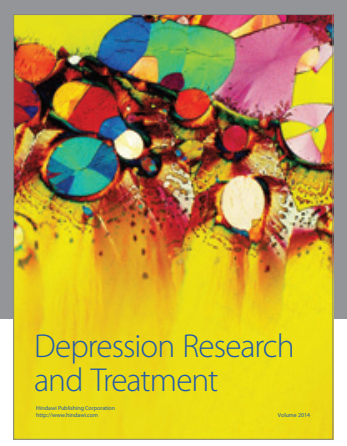
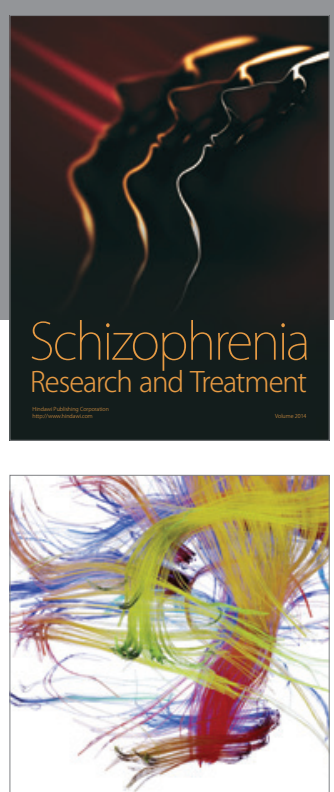

Brain Science

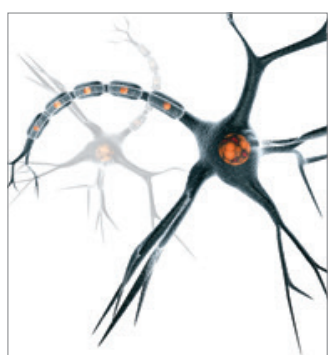

Neural Plasticity
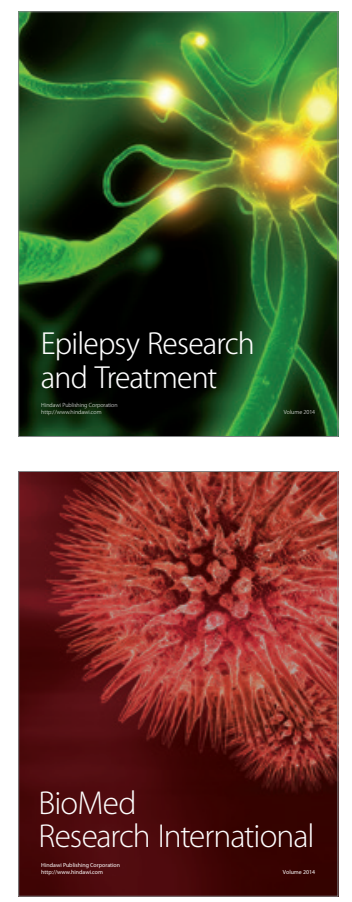

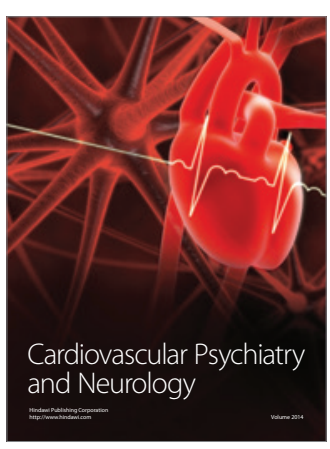

Parkinson's

Disease
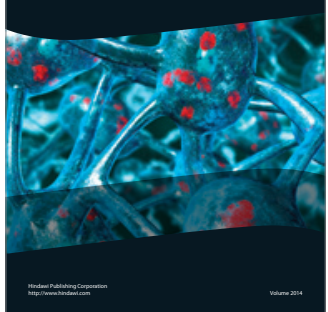